\title{
Traction apophysitis of the olecranon
}

\section{A case report}

\author{
LETHA Y. HUNTER, * M.D., AND GERALD A. O'CONNOR, Ann Arbor, Michigan \\ From the Department of Surgery, Section of Orthopaedic Surgery, University of Michigan, \\ Ann Arbor, Michigan
}

S

everal examples of traction apophysitis occurring in athletic adolescents have been well-described. ${ }^{1-3}$ Little League pitchers can develop medial epicondylitis, jumpers and sprinters may develop pain over their tibial tubercle associated with radiographic evidence of fragmentation of this apophyseal center, and fragmentation of the calcaneal apophysis has been noted in runners. Additionally, the patella, not an apophysis but yet unusual in its location between two strong tendinous structures, was found by Sinding-Larsen ${ }^{4}$ in 1921 to be the site of painful, irregular ossification in athletically active boys from 10 to 14 years of age. This condition affects primarily the lower patellar pole but occasionally is seen at the proximal pole and is associated with swelling of the soft tissues overlying the patella.

In this paper, we describe yet another traction apophysitisthat of the proximal ulnar apophysis in an adolescent diver who placed repeated stress on this growth center by forceful triceps contraction to stabilize his elbow upon entry into the water.

\section{CASE REPORT}

The patient is a 15-year-old right-handed boy who has been diving competitively since he was 6 years old. He has concentrated on $10-\mathrm{m}$ tower dives during the last 6 years. His speed at water impact has been registered at $40 \mathrm{mph}$.

One year ago he began complaining of pain and tenderness associated with swelling over the tip of his right olecranon. The pain increased with severity, especially with tower diving, and decreased with rest. On physical examination he had pain and

\footnotetext{
* Address correspondence to: Letha Y. Hunter, M.D., Sports Medicine Clinic, Hec Edmundson Pavilion (GB-15), University of Washington, Seattle, Washington 98195.
}

swelling over the tip of his right olecranon but had a normal range of motion. Radiographic films of his right elbow demonstrated fragmentation and irregularity of the tip of the proximal apophysis of the right olecranon with overlying soft tissue swelling.

The patient was treated with rest alone, with gradual resolution of pain over a 6- to 8-week period. Following relief of his symptoms, he returned to diving with only occasional symptomatology. His present range of motion remains normal. Repeated radiographs taken at 2, 6, and 10 months demonstrated progressive consolidation (healing) of the avulsed fragment (Fig. 1).

\section{COMMENT}

Because of the high speed at which this young diver hits the water, he needs maximal triceps contraction to stabilize his elbow during head-first entry. Repeated forceful contractions of his triceps presumably placed significant stress on the triceps insertion which resulted in fragmentation of the tip of the olecranon apophysis. This mechanism of injury is reminiscent of the mechanism which resulted in the traction apophysitis of the tibial tubercle, the medial epicondyle, the calcaneus, and the patella.

The only previously reported case of a traction apophysitis of the olecranon we found is in a survey by Francis et al. ${ }^{5}$ of 328 former Little League participants. Of the seven boys demonstrating residual radiographic changes in their throwing arm, one had a small partially reunited avulsion of the olecranon process. No mention was made as to whether or not this boy had been symptomatic during his career in Little League.

The etiology of these traction apophysites remains unclear. Turek $^{6}$ suggested that repeated stresses cause interruption of the blood supply to the involved area. This interruption results in a localized area of avascular necrosis noted radiologically as 

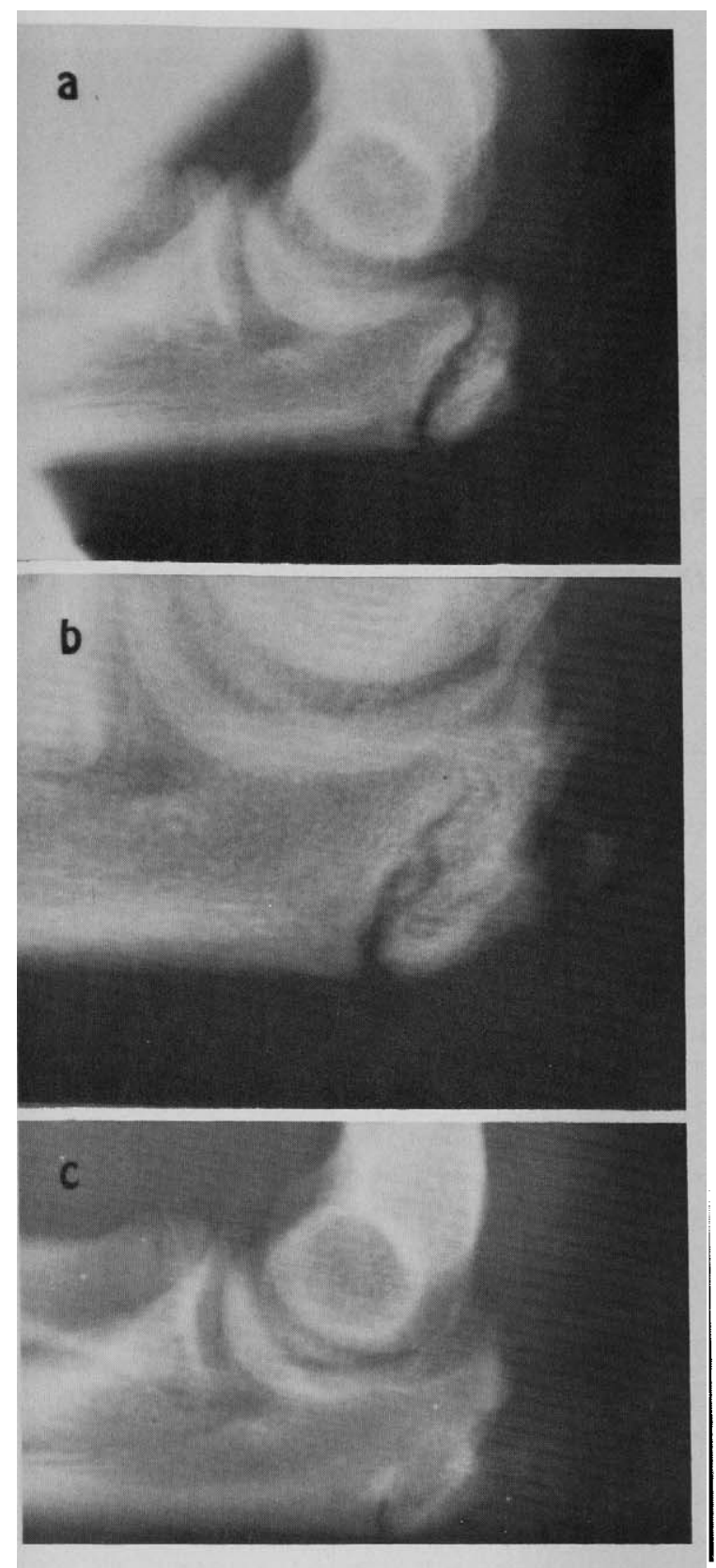

Fig. 1. Radiographs taken at initial visit (a), and at 2 months (b) and 10 months (c) after initial presentation. There appears to be progressive consolidation of an avulsed apophyseal fragment. fragmentation. This lesion may instead be an avulsion fracture of the tip of the apophysis or a partial tearing of the tendinous insertion into the apophysis with resultant calcification. ${ }^{7}$ Fracture through the apophysis has been previously reported in the olecranon, ${ }^{8}$ the medial epicondyle, ${ }^{9}$ and the tibial tubercle. ${ }^{10}$

Symptoms of traction apophysitis typically improve with rest; the only residual is occasionally a prominence of the affected part. No untoward effects have been reported if children with apophysitis of the tibial tubercle or medial epicondyle are allowed to return to their activity when they are symptomatically improved. In our case, consolidation of the fragmentation of the tip of his olecranon was noted radiographically after a period of rest. Consolidation corresponded with clinical improvement.

\section{REFERENCES}

1. Bosworth D: Lesions of the tibial tubercle and their treatment. Am J Surg 43: 526-531, 1939

2. Adams J: Injury to the throwing arm. Calif Med 102: 125-132, 1967

3. Pappas A: The osteochondroses. Pediatr Clin North Am 14: 549 570,1967

4. Sinding-Larsen $\mathrm{M}$ : A hither to unknown affliction of the patella in children. Acta Radiol 1: 171-173, 1921-1922

5. Francis R, Bunch T, Chandler B: Little League elbow: a decade later. Physician Sportsmed 6: 89-94, 1978

6. Turek S: Orihopaedics. Third edition. Philadelphia, JB Lippincott Co, 1977, p 756

7. Medlar R, Lyne E: Sinding-Larsen-Johansson disease. J Bone Joint Surg 60A: 1113-1116, 1978

8. Torg S, Moyer R: Nonunion of a stress fracture through the olecranon epiphyseal plate observed in an adolescent baseball pitcher. J Bone Joint Surg 59A: 264-265, 1977

9. Brogdon B, Crow N; Little leaguer's elbow. Am J Roentgenol 83: $671-674,1960$

10. Hand W, Hand C, Dunn W: Avulsion fractures of the tibial tubercle. $J$ Bone Joint Surg 53A: 1529-1583, 1971 